\title{
Head over heels
}

we explored
the speed
limits of DNA
acrobats
based on
toehold
exchange, and
found that
cartwheeling
makes them
10- to $100-$ fold
faster than
hopping

Dynamic DNA nanotechnology enables the design of DNA-based nanomachines, such as molecular motors or nanorobots. However, most DNA nanomachines operate on a slow timescale, ranging from minutes to hours. Now, Nils Walter and colleagues, writing in Nature Nanotechnology, have made a single-stranded DNA walker that moves by performing cartwheels and at considerably faster speeds than previously reported DNA walkers.

The simplicity and specificity of base pairing make DNA molecules ideal building blocks for the design of nanomachines owing to the ability to programme the hybridization of complementary strands. Most DNA walkers are based on strand displacement, that is, the stepwise replacement of one strand of a double-stranded DNA molecule with a new strand. This reaction is usually catalysed by short overhangs of unpaired nucleotides, called toeholds. The interaction of toeholds with complementary DNA sequences - the footholds - on a surface enables the movement of the DNA machine.

Walter and colleagues designed a single-stranded DNA walker based on such a toehold exchange mechanism that is capable of fast locomotion. "By carefully characterizing bottlenecks in the movement of molecular walkers at the single molecule level, we can design and optimize the speed of autonomous DNA acrobats," says Walter. "In the process, we explored the speed limits of DNA acrobats based on toehold exchange and found that cartwheeling makes them 10 - to 100 -fold faster than hopping."
The cartwheeling locomotion of the DNA acrobat is based on a two-foothold system, two single-stranded DNA toeholds and a flexible linker region. The DNA acrobat then undergoes sequence-guided movement over long distances with one toehold always bound to the complementary foothold. Owing to similar reaction rates, the second toehold rapidly searches for a complementary sequence, binds and thus enables a head-over-heels type of movement. Alternate binding between each of the footholds allows the DNA acrobat to perform an indefinite number of steps over a surface of footholds.

This type of locomotion results in speeds of about $300 \mathrm{~nm}$ per minute with a step size of $7 \mathrm{~nm}$, which was directly measured for individual acrobats using single-molecule fluorescence resonance energy transfer and single-particle tracking. The shorter the length of the toehold, the faster the stepping rate. This design allows the DNA acrobat to cover a distance of over $200 \mathrm{~nm}$ within 10 minutes.

The walk of the DNA acrobat is fast, but random. "Autonomy without the need for any energy input comes at the expense of resulting in a 'random walk' process, which - just like diffusion - is fast at short distances but slows down over long distances owing to the increasing probability to also take steps back," explains Walter.

To overcome this limitation, the researchers envisage coupling some form of energy consumption process to make the DNA acrobat move in a specific direction. One approach to achieve directional motion could be a recently

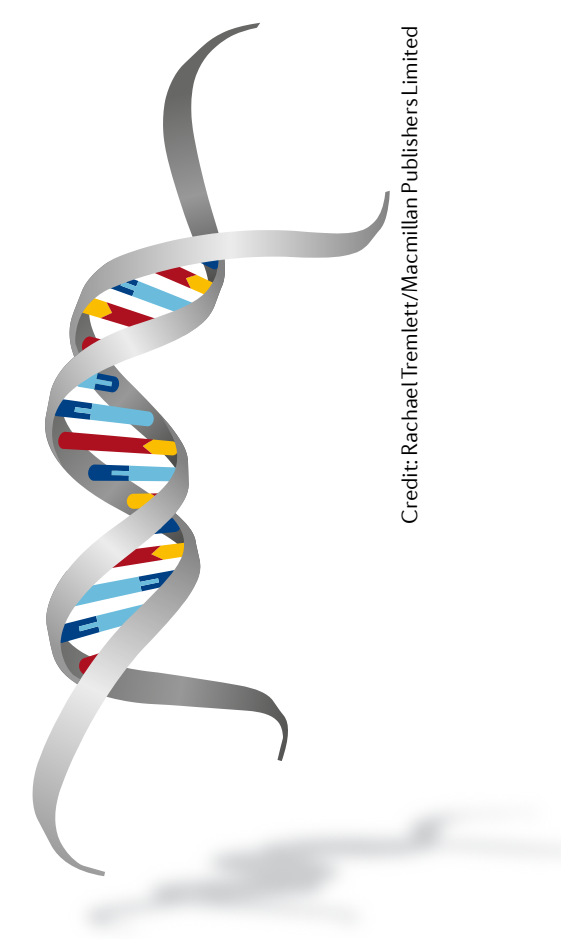

developed bio-hybrid nanoengine. This nanoengine uses a catalytic stator that unidirectionally rotates an interlocked doublestranded DNA wheel, powered by the hydrolysis of nucleotide triphosphates (NTPs), with a T7 RNA polymerase as the power generator. Such NTP-driven locomotion enables directional movement and, thus, could be combined with the DNA acrobat to further increase its speed and to endow the acrobat with directionality.

Christine-Maria Horejs

ORIGINAL ARTICLES Li, J. et al. Exploring the speed limit of toehold exchange with a cartwheeling DNA acrobat. Nat. Nanotechnol. https://doi.org/10.1038/s41565-018-0130-2 (2018) | Valero, J. et al. A bio-hybrid DNA rotor-stator nanoengine that moves along predefined tracks. Nat. Nanotechnol. https://doi.org/10.1038/ s41565-018-0109-z (2018) 